\title{
Correction to: Filtering Techniques for Regular Expression Matching in Strings
}

Tao Qiu, Xiaochun Yang, and Bin Wang

\section{Correction to:}

Chapter "Filtering Techniques for Regular Expression Matching in Strings" in: C. Liu et al. (Eds.): Database Systems

for Advanced Applications, LNCS 10829, https://doi.org/10.1007/978-3-319-91455-8_12

The original version of the chapter starting on p. 118 was revised.

The title of the chapter has been supplemented with "Invited Talk".

The acknowledgement "This paper constituted an invited talk, held at BDQM 2018, a DASFAA 2018 satellite workshop. The main techniques derive from our work cited in [11]" has been added.

The original chapter was corrected. 